\title{
MANUFACTURING OF DEVICES FOR THE PARALLEL PRECISION ALIGNMENT OF MULTIPLE MICRO COMPONENTS
}

\author{
Christian Brecher, Martin Weinzierl
}

Fraunhofer Institute for Production Technology IPT, Steinbachstraße 17, 52074 Aachen

martin.weinzierl@ipt.fraunhofer.de

\begin{abstract}
Micro components are available in a variety of shapes typically sizing from $1 \mathrm{~mm}$ down to $0.01 \mathrm{~mm}$. Their mass production is quite common using state of the art production technology. Micro spheres for example, are on the one hand available in lot sizes up to some thousands in a constant quality within micron accuracy [1]. On the other hand they are quite commonly provided as bulk material with diameter variations of up to $10 \%$ in each lot size [2]. In both cases, the bulk micro components are usually arranged in incoherent batches which are packed in plastic bags or small jars for handling and shipping. The decollating of single components for follow-up micro assembly processes is complicated by the wellknown effects in micro handling such as dominating adhesion and friction forces [3]. These effects limit the post processing of bulk micro components to manual work in order to sort and align the single micro components prior to their exposure to an automated assembly line. To enable a sophisticated and automated handling of the single micro components, automated sorting and alignment mechanisms are necessary to arrange the bulk micro components in a well defined pattern structure which is essential to achieving an efficient automated micro assembly.
\end{abstract}

\section{Development of passive alignment structures}

In preceding researches of the Collaborate Research Centre SFB 440, passive alignment structures have been designed for elementary geometrical structures such as spheres, cubes and cylinders [4]. For each of those geometrical elements, statically determinant structures have been designed and optimised regarding the positioning accuracy of the micro components. As those researches have shown, passive alignment allows for positioning accuracies down to the single digit micrometer range. However, in these investigations each micro component has been handled and aligned manually. The manual alignment of the single micro components prior to their exposure to an automated assembly processes is state of the art in micro production today but reveals a gap in the fully automated process chain ranging from the bulk production of components to the finished micro products. For an efficient production of micro systems, multiple components have

Please use the following format when citing this chapter:

Brecher, C., Weinzierl, M., 2008, in IFIP International Federation for Information Processing, Volume 260, MicroAssembly Technologies and Applications, eds. Ratchev, S., Koclemeijer, S., (Boston: Springer), pp. 297-304. 
to be handled and aligned with a reproducible precision. In the current research work which is conducted at the Fraunhofer IPT, mechanisms for the simultaneous alignment of multiple micro components are developed to bridge the gap between the automated bulk production of micro components and the automated assembly of micro systems. The bases for these mechanisms are arrays composed out of passive alignment structures and the use of active energies, e.g. airflow, which support the alignment of the micro components in these arrays. Such arrays have been realised for the parallel alignment of micro spheres as well as cubical and cylindrical micro components. Due to the widely spread application of micro spheres in micro assembly, the development of array structures for passively aligning an unsorted bulk of micro spheres is introduced in the following.

\subsection{Array structures for the parallel passive alignment of micro spheres}

The design of the passive alignment array structures is based on the fundamental results from the investigations on passively aligning single spheres where flat, round and pointed elements have been deployed and analysed regarding their alignment accuracies. Here, three-sided pyramid cavities not only provide a simple solution to realise a statically determined three point contact but also have been proved to be least susceptible to misalignment due to form tolerances of the structure and micro sphere. Another essential element which influences the design of the array structures is the ability for the rapid manufacturing by ultraprecision machining and replication through hot embossing [5]. The combination of ultraprecision machining and replication processes like hot embossing not only has a great potential for low cost mass production but also enables the manufacturing of passive alignment concepts which cannot be machined directly by conventional cutting processes. A good example for this is the realisation of the array concepts which is introduced in this paper. These passive alignment arrays are designed in such a way that they are composed of linear elements like intersecting bridges (Figure 1). The tools which are used for the hot embossing of these arrays feature column structures which are formed by intersecting grooves which can be manufactured very efficiently within a few hours of time by ultraprecision machining with accuracies down to the submicron range. 


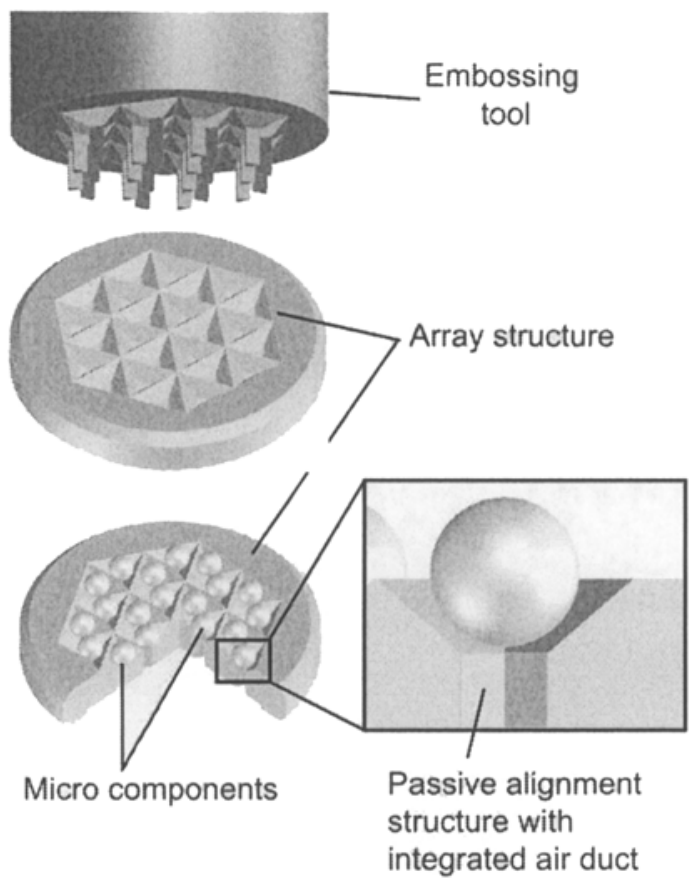

Fig. 1. Concept for ultraprecision machined embossing tools for the replication of passive alignment array structures

\section{Ultraprecision machining of passive alignment structures}

In ultraprecision machining, conventional metal cutting processes such as turning and milling, are used to manufacture micro structures with form accuracies down to $0.1 \mathrm{~mm} / 100 \mathrm{~mm}$ and an optical surface finish with an average roughness $<10 \mathrm{~nm}$ Ra. The classical application of ultraprecision machining is the manufacturing of complex master tools for optical applications such as reflectors or display components (Figure 2). Due to the high form accuracy and surface finish which can be realised by ultraprecision machining, this manufacturing process is advanced at Fraunhofer IPT to manufacture high precision micro assembly devices such as vacuum grippers and passive alignment structures.

The extremely high form accuracies and the optical surface finish is mainly achieved by using ultraprecision machine tools and single crystal diamond cutting tools. Ultraprecision machine tools mainly differ from conventional machine tools in the machine bases, drives, guides and spindles which are used. As a material for the machine base, solid granite is used to achieve high thermal stability as well as excellent damping properties. Minimum step sizes below $50 \mathrm{~nm}$ are realised with the use of highly dynamic direct drives in combination with high precision linear glass scales which allow for an interpolated resolution in the single digit nanometer 
range. The guidances of the feed and positioning axes as well as the spindle rotors are designed with hydro- or aerostatic supports. These allow for a contact less support of the moving parts and therefore eliminate the so-called "stick slip" effect which reduces the positioning accuracy of conventional ball bearing supported machine tools to a couple of microns. Due to this, the single axes of ultraprecision machine tools can be moved extremely smoothly within nanometer steps. This results in sub-micron form accuracies and optical surfaces without requiring any further finishing.

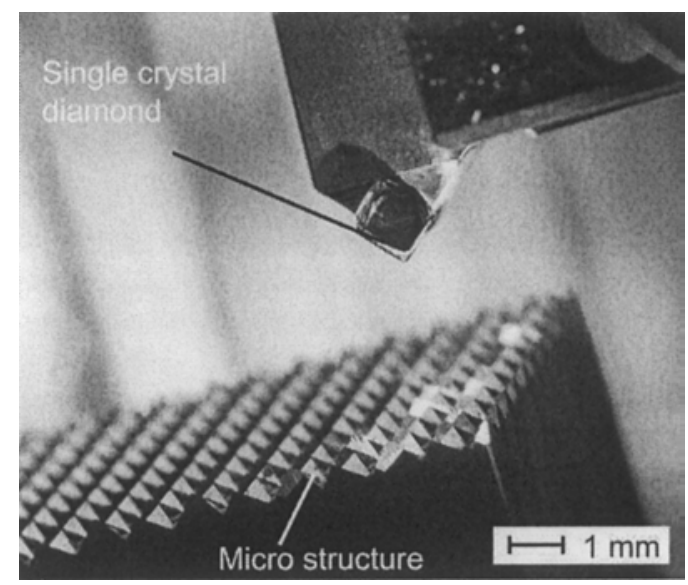

Fig. 2. Ultraprecision machining using single crystal diamond tools

The single crystal diamond tools which are mainly responsible for realising the optical surface finish of ultraprecision machined work pieces are available in a great variety of shapes such as v-groove-, radius- or facets. Their most significant feature is an extremely sharp cutting edge with an edge radius $<50 \mathrm{~nm}$. These sharp cutting edges as well as the generally low friction coefficient of diamond enable a submicron chip removal and very low cutting forces which minimizes tool chattering and tool deflection during the machining process. In many application of ultraprecision machining the geometry of the micro structure is a projection of the diamond tool geometry. This requires an absolutely notch-free cutting edge since the smallest irregularities in the surface flanks can disturb the function of the work piece. In optical applications, score marks on the work piece surface cause diffraction patterns. In the case of passive alignment structures, such score marks cause a misalignment of the micro parts.

\subsection{Geometric variety of ultraprecision machined micro structures}

By combining different ultraprecision machining processes such as turning, flycutting or planing and using varying shapes of diamond tools, a great variety of micro structures can be cut into non iron metals or nickel coated steel bodies. In ultraprecision machining, linear groove structures are usually manufactured by flycutting or planing. Fly-cutting is a milling process using one single diamond tool 
which is revolving on an aerostatic supported rotor of a high precision spindle. This rotating tool is slowly moved across the work piece surface with a maximum infeed up to $2 \mathrm{~mm}$. In planing processes, the tool is mounted in a fixed position on a highly dynamic feed axis and is moved across the work piece surface with up to $60.000 \mathrm{~mm} / \mathrm{min}$ at maximum in feeds below $10 \mu \mathrm{m}$. In both cases a groove is cut into the work piece surface which corresponds exactly to the cutting edge of the diamond tool. By machining intersecting grooves in different directions and applying different tool geometries, a great variety of complex micro structures can be manufactured. The decision which ultraprecision machining process is applied usually depends on the properties of the micro structure and the resultant machining time. Furthermore, fly-cutting has the advantage of far lower tool wear compared to planing. The process-related cinematic roughness in fly-cutting can be reduced down to the sub-nanometer range by optimally adjusting the feed rate to the spindle revolution and can thus be considered as negligible. Therefore, fly-cutting is well suitable for the machining of high aspect ratio micro structures with sub micron form accuracies as they are required for the embossing tools for the passive alignment arrays.

\section{Hot embossing of passive alignment structures}

Within the Collaborate Research Centre SFB 440, hot embossing is used to manufacture passive alignment structures by replication. During hot embossing, a thermoplastic substrate is softened plastically by heating in an evacuated chamber. Subsequently, the micro structured embossing tool is slowly pressed into the molten substrate. After cooling down, the substrate keeps the imprint of the embossing tool which is withdrawn when the substrate has solidified.

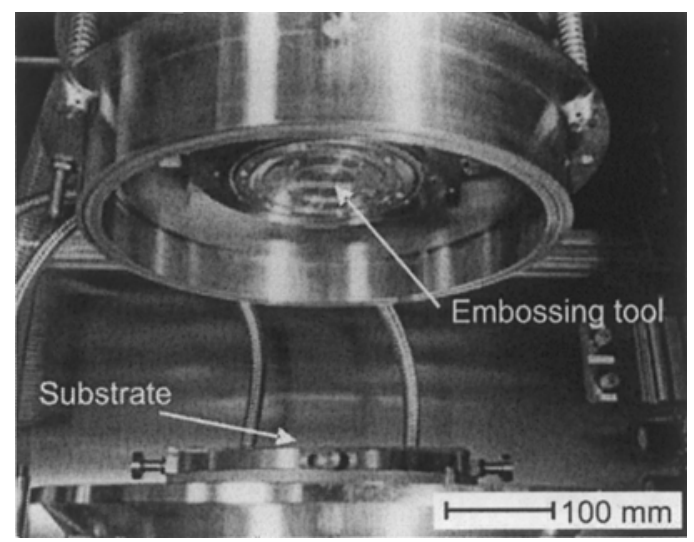

Fig. 3. Embossing chamber of a Jenoptik HEX02 hot embossing machine 
In contrast to other replication processes, e.g. injection molding, the substrate yields very little which leaves almost no inner tension in the replica. Therefore hot embossing is especially suitable for the highly accurate replication of micro structures. The quality of the replicated micro structures is directly dependent on the quality of the embossing tool. Therefore, the form tolerances and surface finish which are achieved in the manufacturing of the embossing tool are decisive for the replicated micro structures.

\section{Manufacturing and practical testing of the passive alignment arrays}

The machining of the appropriate embossing tool requires a considerable effort for set up and referencing the tool and the work piece. Since the three-sided cavities and channels are the negative imprint of triangular column structures, the ultraprecision machining process has to be set up in such a way that the grooves which are machined by fly-cutting from three different directions (included angle: $60^{\circ}$ ) cross in exactly one point at each intersection. Otherwise, artifacts would remain between the cavities which would decrease the functionality of the array. Therefore, three grooves which intersect by $60^{\circ}$ have been machined in sample cuts which have been analysed by optical microscopy prior to the machining of the actual structure. By measuring the offset between two intersecting grooves and the remaining groove, correction values have been determined to position the tool in such a way that all tree direction intersect within one point with an accuracy $<0.5 \mu \mathrm{m}$. This set-up in combination with an appropriately shaped diamond tool enables the manufacturing of regular three-sided pyramids with straight columns on top (Figure 5). The imprint of these columns form funnels which merge into squared channels which serve as air ducts to suck in the micro spheres.

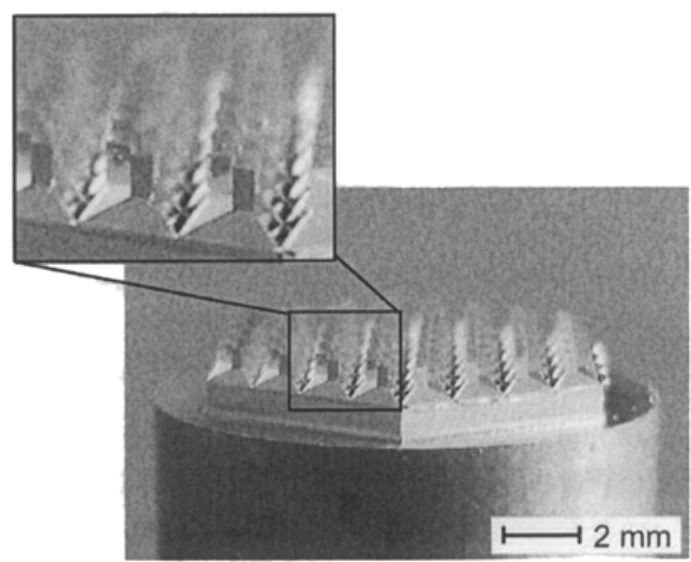

Fig. 4. Embossing tool with three sided column structures 
Each cavity can hold exactly one micro part in a defined position. When the array is connected to a suction gripper and positioned over an incoherent batch of micro components the air flow sucks the micro components into the cavities which are then plugged and the air flow is cut off. When the array is filled up, excessive micro components fall off or are not even grasped in the first place. In this way, a defined quantity of micro components can be picked up and is aligned with a defined and constant pitch simultaneously (Figure 6). The symmetry of those micro components supports their self-alignment in the single cavities of the array structures.
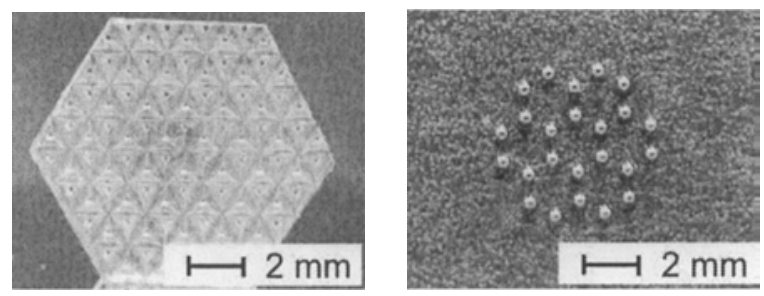

Fig. 5. Micro sphere-array (left) and aligned micro spheres (right)

\section{Outlook}

The passive alignment arrays are suitable for the secure handling and shipping of the sorted micro components as well. For this purpose, a cap with clip elements has been designed, in which the array can be locked in place. A soft inlay at the bottom of the cap adapts to the shape of the micro components and holds them in position when the array is released from the suction gripper. An adequate pick-up and alignment mechanism can then be realized as follows (Figure 7):

1. Gripping the array with a vacuum gripper

2. Sucking the micro spheres into the cavities of the array

3. Engaging the array into the cap

4. Cutting off the air flow and removing the suction gripper

This mechanism enables the alignment and handling of a fixed quantity of micro spheres in a defined position. Optionally, the arrays can be used in the same way to assort a loose batch of micro spheres right before an automated micro assembly line. Due to their regular three sided passive alignment features, the arrays can cope with varying diameters and still keep up a lateral positioning of the micro spheres at constant distances. The upcoming research activities will focus on the realization of the pick-up and alignment mechanism as described above. With the aid of active energies such as air flow and vibration, the fully automated alignment and temporary fixation of a loose batch of micro spheres will be realised. 

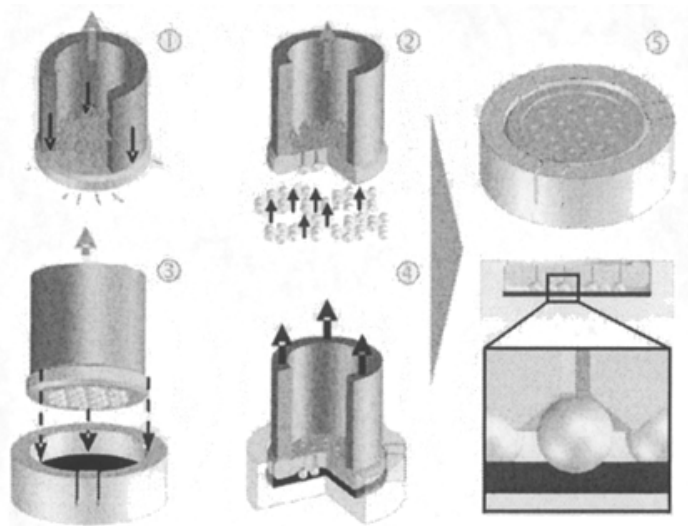

Fig. 6. Mechanism fort the parallel alignment and fixation of micro spheres

Furthermore, the machining of three dimensionally arranged passive alignment structures as well as the use of passive alignment structures to support high precision joining processes will be investigated within the Collaborate Research Centre SFB 440. Therefore the advanced design of passive alignment micro structures will aim for providing a fixed reference in the joining layer to prevent the shape distortion of micro components during gluing or brazing.

\section{Acknowledgements}

The authors would like to thank the German Research Foundation (DFG) for their support within the Collaborate Research Centre SFB 440. Through their funding of the sub-project A7 $\gg$ Assembly Supporting Mechanical Structures for the Passive Alignment of Micro Components« the DFG has enabled the research activities described above.

\section{References}

\section{ISO 3290 / DIN 5401}

2. Brandau, T., Mikroverkapselte Wirkstoffe und Mikrokugeln in pharmazeutischen und kosmetischen Anwendungen, Chemie Ingenieur Technik 75, No. 11, Weinheim, 2003, pp. $1741-1745$

3. Petersen, B., Flexible Handhabungstechnik für die automatisierte Mikromontage, Dissertation RWTH-Aachen, Aachen, 2003, pp. 12 - 14

4. Brecher, C.; Weinzierl, M.; Lange, S., Development of Passive Alignment Techniques for the Assembly of Hybrid Micro systems, Precision Assembly Technologies for Mini and Micro Products, Proceedings of the IFIP 12, Springer Verlag New York, USA 2006, pp. 611-615, ISBN 0-387-31276-5

5. Brecher, C.; Weinzierl, M.; Lange, S.; Peschke, C., Active and Passive Tool Alignment in Ultraprecision Machining for the Manufacturing of Highly Precise Structures, Production Engineering - Research and Development, Annals of the German Academic Society for Production Engineering WGP 8 (2006), XIII/1, pp. 193 - 196 